Artigo Original

Original Article

Luciana Mendonça Alves ${ }^{1,2}$ (C)

Luiz Felipe dos Santos ${ }^{1}$ (1)

Izabel Cristina Campolina Miranda ${ }^{1}$ (B)

Isa Mourão Carvalho ${ }^{1}$

Gabriela de Lima Ribeiro' ${ }^{1}$ (B)

Laura de Souza Cardoso Freire' ${ }^{1}$

Vanessa de Oliveira Martins-Reis ${ }^{3}$ (1)

Letícia Correa Celeste ${ }^{3,4}$

Descritores

Linguagem

Leitura

Escolaridade

Estudantes

Aprendizagem

Keywords

Language

Reading

Educational Status

Students

Learning

Endereço para correspondência:

Luciana Mendonça Alves

Departamento de Fonoaudiologia,

Faculdade de Medicina, Universidade

Federal de Minas Gerais - UFMG

Av. Prof. Alfredo Balena, 190,

Belo Horizonte (MG), Brasil,

CEP: 31270-901.

E-mail: lumendoncaalves@gmail.com

Recebido em: Junho 09, 2020

Aceito em: Setembro 24, 2020

\section{Evolução da velocidade de leitura no Ensino Fundamental I e II}

\author{
Reading speed in Elementary School and \\ Junior High
}

\section{RESUMO}

Objetivo: Verificar a influência da escolaridade em medidas de velocidade de leitura no ensino fundamental e explorar os níveis de precisão das variáveis da velocidade de leitura nos estágios de investigação epidemiológica de casos. Método: Participaram 535 escolares do $2^{\circ}$ ao $9^{\circ}$ ano de escola particular e pública. A leitura oral de um texto simples foi avaliada nos parâmetros palavras lidas por minuto e palavras lidas corretamente por minuto. Foram conduzidas medidas de estatística descritiva e teste $\mathrm{T}$ de student com significância de $5 \%$, além do escore Z, padronizado e individual. Resultados: Os resultados corroboram a literatura que evidencia aumento da velocidade de leitura com a progressão da escolaridade. A fluência leitora se mostrou em processo de construção entre o $2^{\circ}$ e o $7^{\circ}$ ano do Ensino Fundamental, com estabilização a partir do $7^{\circ}$ ano. Uma leitura mais fluente e homogênea se mostrou sedimentada entre o $7^{\circ}$ e o $9^{\circ}$ ano. O estudo propõe a interpretação dos achados com escalas sugestivas para déficit por meio do z-escore. Os dados são estratificados desde valores sugestivos de déficit importante até o não sugestivo, com um total de cinco estratos. A estratificação permite uma referência mais clara sobre qual ponto se encontra o escolar nas faixas esperadas para sua escolaridade. Conclusão: As medidas dos valores esperados segundo a escolaridade são essenciais para melhor conhecimento do desenvolvimento da leitura, de forma a prover padrões de referência para um adequado monitoramento no âmbito clínico e educacional e predição das habilidades e dificuldades leitoras.

\begin{abstract}
Purpose: To verify the influence of schooling on reading speed measures in Elementary School and Junior High, also aims to explore the levels of accuracy of the variables of reading speed in the epidemiological investigation stages of cases. Methods: 535 students from the 2 nd to the 9 th grade from a private school and a state school system participated in the study. The oral reading of a simple text was evaluated in the following parameters: words read per minute and correctly words read per minute. Descriptive statistics and T-test measures with the significance of $5 \%$ were conducted, besides the z-score, standardized and individual. Results: The results corroborate the literature review which shows an increase in reading speed with the progression of schooling. The reading fluency was presented in the construction process between the 2 nd and 7 th grades, with stabilization from the 7th year. A more fluent and homogeneous reading was sedimented between the 7th and 9th grades The study proposes the interpretation of the findings with scales suggestive of the deficit using the z-score. The data are stratified from values suggestive of significant deficit to non-suggestive, with a total of five strata. The stratification allows a clearer reference on which point the scholar found in the expected ranges for their schooling. Conclusion: The measures of the expected values according to schooling are essential for better-quality knowledge of reading development, to provide reference standards for adequate monitoring in the clinical and educational sphere and prediction of reading skills and difficulties.
\end{abstract}

Trabalho realizado na Universidade Federal de Minas Gerais - UFMG - Belo Horizonte (MG), Brasil

${ }^{1}$ Departamento de Fonoaudiologia, Faculdade de Medicina, Universidade Federal de Minas Gerais - UFMG Belo Horizonte (MG), Brasil.

${ }^{2}$ Programa de Pós-graduação em Ciências Fonoaudiológicas, Faculdade de Medicina, Universidade Federal de Minas Gerais - UFMG - Belo Horizonte (MG), Brasil.

${ }^{3}$ Curso de graduação em Fonoaudiologia, Universidade de Brasília - UnB - Brasília (DF), Brasil.

${ }^{4}$ Programa de Pós-graduação em Ciências da Reabilitação, Universidade de Brasília - UnB - Brasília (DF), Brasil.

Fonte de financiamento: nada a declarar.

Conflito de interesses: nada a declarar.

(c) (i) Este é um artigo publicado em acesso aberto (Open Access) sob a licença Creative Commons Attribution, que permite uso, distribuição e reprodução em qualquer meio, sem restrições desde que o trabalho original seja corretamente citado. 


\section{INTRODUÇÃO}

A capacidade de compreender o que é lido tem sido fortemente relacionada ao desenvolvimento da precisão do reconhecimento de palavras e da fluência da leitura ${ }^{(1-6)}$. Com o passar dos anos escolares, a velocidade com a qual o estudante lê aumenta, como mostram alguns estudos ${ }^{(3,5,6)}$. Entretanto, algumas perguntas importantes ainda precisam ser respondidas em relação à população de escolares do Brasil: quais os valores esperados de velocidade de leitura em cada série escolar e quando se deve levantar suspeita de dificuldades de fluência de leitura? E ainda, com o avançar dos anos escolares, em qual momento ocorrerá a estabilização do aumento da velocidade de leitura, aproximando-se dos padrões do adulto fluente?

Quando os alunos começam a ler, seus esforços em decodificação consomem a maioria de seus recursos atencionais e suas leituras serão inicialmente mais lentas e com muito esforço. À medida que aumenta a familiaridade com as palavras escritas, a taxa de leitura aumenta, assim como a fluência ${ }^{(1-5)}$.

A fluência leitora é a habilidade de ler de forma precisa com velocidade natural e expressividade ${ }^{(7)}$ e combina acurácia, automaticidade e expressividade ${ }^{(8)}$.

A acurácia refere-se à decodificação correta das palavras combinando um forte entendimento do princípio alfabético, a destreza para juntar diferentes sons e o conhecimento de um grande número de palavras. Deste ponto, se estabelece a automaticidade na leitura, que é constituída por quatro elementos: velocidade, ausência de esforço, autonomia e ausência de atenção consciente ${ }^{(9,10)}$.

Logo que a decodificação de palavras é alcançada e se torna sem esforço e rápida, a leitura eficiente do texto se expressa com prosódia adequada, ou seja, a leitura passa a ter expressão, ritmo e entonação apropriados, permitindo a manutenção do significado $^{(8,11)}$

É reconhecido que a fluência é um componente crítico da leitura hábil e seu desenvolvimento pode ser monitorado acompanhando a acurácia na leitura, medida pela contagem de palavras lidas de forma correta, excluindo-se aquelas lidas incorretamente, com substituições, omissões ou inserções. Pode ser monitorada também pela velocidade de leitura, quantidade de palavras que se consegue ler em determinado tempo, além das variações prosódicas durante a leitura em voz alta ${ }^{(1,12-14)}$.

Ferramentas de diagnóstico da educação básica nacional, tais como o IDEB (Índice de Desenvolvimento da Educação Básica) mostraram que em 2017 o Brasil não atingiu resultados significativos na área educacional. Somente os anos iniciais do fundamental conseguiram cumprir a meta de 5,8. Os anos finais do ensino fundamental obtiveram média nacional de 4,7, posto que a meta estabelecida era de 5 pontos. No ensino médio, o país alcançou 3,8 , ficando muito aquém do esperado ${ }^{(15)}$. Os dados apresentados por essa aferição evidenciam que alunos ao final da educação básica apresentam médias baixas. Posto isso, o monitoramento sistemático da leitura em sala de aula pode se constituir em uma importante estratégia de identificação precoce de escolares em situação de risco, permitindo a adoção de metodologias educacionais mais assertivas e intervenções remediadoras, antes que o progresso acadêmico seja comprometido.

Outro ponto importante a salientar é a falta de estudos nacionais que investiguem o desempenho esperado e estabeleçam parâmetros para a avaliação da leitura e da escrita para cada série escolar ${ }^{(16,17)}$.
Com isso, torna-se necessário conhecer o processo de evolução da fluência leitora nos estudantes brasileiros, a fim de gerar parâmetros de monitoramento do desenvolvimento da leitura ao longo dos ciclos escolares. Neste sentido, estudos demonstram que a fluência leitora dos escolares aumenta conforme avança a escolaridade ${ }^{(16-19)}$

Considerando-se a importância da leitura para garantir a participação e autonomia na sociedade e o impacto do seu desempenho na vida acadêmica e pessoal, o presente estudo busca verificar a influência da escolaridade em medidas de velocidade de leitura no ensino fundamental e explorar os níveis de precisão das variáveis da velocidade leitora nos estágios de investigação epidemiológica de casos.

\section{MÉTODO}

Trata-se de estudo observacional, analítico transversal aprovado pelo Comitê de Ética em Pesquisa sob número de protocolo CAAE 38861914.4.0000.5096 e parecer n ${ }^{\circ} 885546$. Todos os responsáveis pelos participantes assinaram o termo de consentimento livre e esclarecido (TCLE), e os escolares participantes assinaram o termo de assentimento livre e esclarecido.

Para a análise da velocidade de leitura, foram realizadas gravações em áudio da leitura em voz alta e de forma natural de um texto de nível fácil, contendo 210 palavras ${ }^{(20)}$. Para cálculo amostral foi considerado erro de $5 \%$, índice de confiança de $95 \%$ e foram utilizadas informações de 2018 do IBGE $^{(21)}$ para a cidade de Belo Horizonte, com resultado de 174 estudantes para o ensino fundamental. Entretanto, como a escolha das escolas não foi aleatória, participaram as duas escolas que aceitaram e extrapolamos o número sugerido em ambas as redes: pública e privada, totalizando 535 escolares do $2^{\circ}$ ao $9^{\circ}$ ano do Ensino Fundamental de duas escolas da região metropolitana de Belo Horizonte, Minas Gerais. Destes, 180 alunos eram da escola da rede privada e 355 da rede pública de ensino, totalizando 259 alunos do Ensino fundamental I e 276 no Fundamental II.

A escolha de escolas se deu com base na semelhança dos índices de educação que exibem as instituições pesquisadas, pela diversidade do ensino e para garantir que os resultados obtidos na pesquisa possuíssem uma maior validade externa para a amostra. Realizar comparações entre os sistemas de ensino não foi um dos objetivos desta pesquisa. As duas escolas apresentaram Ideb (Índice de Desenvolvimento da Educação Básica) em torno de 7 (6,9 e 7,2) e Saeb (Sistema de Avaliação da Educação Básica) de 7,55 e 7,53 (dados disponíveis no site do INEP- Instituto Nacional de Estudos e Pesquisas Educacionais Anísio Teixeira) $)^{(15)}$. São escolas localizadas em regiões mais centrais da cidade, com perfis sociodemográficos similares. Não se trata de populações em situação de vulnerabilidade.

Para participarem da pesquisa os alunos deveriam estar matriculados do $2^{\circ}$ ao $9^{\circ}$ ano do Ensino Fundamental em uma das escolas mencionadas, sem distorção série-idade e diagnóstico de alterações de aprendizagem, linguagem, problemas sensoriais ou cognitivos. Os escolares com tais diagnósticos foram excluídos para evitar possíveis excessos de outliers na análise estatística. Tais informações para inclusão na pesquisa foram colhidas via questionário anexo ao TCLE respondido pelos pais, coordenadores pedagógicos e professores. Os escolares participantes e seus responsáveis legais foram esclarecidos sobre os objetivos, riscos e 
benefícios da pesquisa. Aqueles que não preencheram os critérios descritos, que não concordaram em participar ou que os pais não assinaram o TCLE foram excluídos. Assim, foram excluídos 74 participantes por tais critérios de exclusão.

A coleta de dados aconteceu ao final do segundo semestre, entre os meses de maio e julho. Foi utilizado um computador portátil conectado a um microfone unidirecional de cabeça e as gravações da leitura em voz alta dos participantes foram realizadas no software Praat ${ }^{(22)}$. O procedimento foi realizado individualmente, em sala isolada e sem interferência sonora do ambiente externo dentro das próprias escolas, em contraturno ao horário das aulas, de forma a não comprometer a aprendizagem do conteúdo curricular estabelecido pelas instituições de ensino.

Antes de iniciarem a leitura foram esclarecidas eventuais dúvidas dos estudantes sobre a atividade a ser executada. Após a leitura, os participantes responderam a um questionário, de múltipla escolha, com dez perguntas sobre o texto lido $^{(20)}$. Ressalta-se que o objetivo da pesquisa não era o de testar a compreensão. Tal recurso foi utilizado apenas para constatar se havia leitura atenta e capaz de evocar fatos literais e inferenciais do texto e não uma decodificação automática, sem acesso ao significado.

Foram avaliados os seguintes parâmetros nas gravações da leitura: palavras lidas por minuto (PPM) - taxa de fluência - e palavras lidas corretamente por minuto (PCPM) - acurácia. Para a análise de PPM, todas as gravações realizadas foram ouvidas e foi contabilizado o número total de palavras lidas no texto completo. Destas foram consideradas somente as palavras lidas corretamente para calcular as PCPM. Essas análises foram feitas por pessoas distintas, a fim de proporcionar maior fidedignidade ao trabalho ou seja, conferência das medidas realizadas.

As variáveis sexo e idade não foram consideradas para análise neste estudo. Primeiramente, não foi objetivo desta pesquisa realizar comparação entre sexos, uma vez que estudo realizado na população brasileira não evidenciou distinção significativa no desempenho em tais habilidades entre os meninos e meninas ${ }^{(17)}$. E ainda, não foi objeto analisar a idade, mas sim entender a evolução das medidas realizadas no decorrer dos anos escolares. Como na amostra não há distorção idade-série, e no Brasil há critérios rígidos quanto à idade da criança em cada série, podemos considerar que a escolaridade reflete o desempenho da idade.

Para atingir o objetivo da pesquisa de avaliar a evolução da velocidade de leitura de alunos do $2^{\circ}$ ao $9^{\circ}$ ano do Ensino Fundamental, foi importante garantir que o texto lido assegurasse a boa capacidade de decodificação e reconhecimento automático das palavras, como forma de disponibilizar recursos cognitivos para o acesso aos padrões de fluência tanto aos alunos do Ensino Fundamental I quanto aos do Ensino Fundamental II. Por isso foi oferecido o texto já bem referenciado na literatura (20) com extensão e grau de complexidade confortáveis para que os escolares pudessem focar nos aspectos de fluência e não concentrassem esforços cognitivos na habilidade de decodificação. Tal metodologia baseou-se em estudo já realizado no Brasil com população do Ensino Fundamental II ${ }^{(17)}$.

As taxas de fluência e acurácia para cada leitor foram calculadas a partir da metodologia explicada a seguir ${ }^{(12)}$.

A fórmula utilizada para calcular as palavras lidas por minuto (PPM) foi a seguinte:

$P P M=\frac{\text { Número de palavras lidas } \times 60 \text { segundos }}{\text { tempo total de leitura }(\text { em segundos })}$
Para o cálculo de palavras lidas corretamente por minuto (PCPM) estimou-se o número de palavras lidas corretamente, considerando apenas as palavras que foram lidas de forma correta e fluente. Foram considerados erros de leitura a falha na decodificação, omissões e hesitações, erro quanto ao emprego da tonicidade e desrespeito ao sinal gráfico de acentuação. O cálculo para esse quesito se deu usando a seguinte fórmula:

$P C P M=\frac{\text { Número de palavras lidas corretamente } \times 60 \text { segundos }}{\text { tempo total de leitura }(\text { em segundos })}$

Os dados foram analisados por meio de estatística descritiva (média, mediana e desvio padrão) e teste de normalidade de Anderson-Darling com distribuição normal dos dados. Para comparação das variáveis de velocidade de leitura entre os anos escolares foi utilizado o teste $t$ de student para amostras não pareadas para cada ano escolar. O nível de significância adotado foi de $5 \%$.

A fim de delimitar as faixas relativas a cada ano escolar estudado, foi utilizado o escore Z, padronizado e individual, com a seguinte fórmula:

$\frac{Z=X-\bar{X}}{d p}$

Na qual,

$X=$ escore bruto

$\bar{X}=$ média do grupo no teste.

$d p=$ desvio-padrão do grupo normativo no teste.

Para análise epidemiológica de sugestivos de déficits, a seguinte pontuação foi utilizada ${ }^{(23)}$ :

$Z \geq-1,0$ desvio padrão - não sugestivo de déficit

$Z$ entre - 1,0 e - 1,5 desvio padrão - sugestivo de alerta para déficit

Z entre - 1,6 desvio-padrão e - 2,0 desvio-padrão - sugestivo de déficit moderado à severo.

$\mathrm{Z} \leq-2,0$ desvios-padrão - sugestivo de déficit de gravidade importante.

\section{RESULTADOS}

A Tabela 1 apresenta os valores de média, desvio padrão e coeficiente de variação obtidos para cada ano escolar referentes a PPM, PCPM e tempo total de leitura, na qual se observa que as medidas de fluência (PPM) e acurácia (PCPM) foram aumentando do segundo ao nono ano do Ensino Fundamental. Na Figura 1 fica mais fácil visualizar o coeficiente de variação de cada ao escolar, observando-se diminuição com o aumento dos anos escolares. Isso indica que nas séries iniciais a variabilidade de desempenho em fluência de leitura entre as crianças é muito grande enquanto que ao final do Ensino Fundamental observa-se uma homogeneidade no desempenho.

Os resultados da comparação entre os anos escolares, indicados na Tabela 2, apontam que a progressão tem diferença estatisticamente significativa entre o $2^{\circ}$ e o $7^{\circ}$ ano. Entre o $7^{\circ}$ e o $8^{\circ}$ e entre o $8^{\circ}$ e o $9^{\circ}$ ano não foram encontradas diferenças significativas. Entretanto, a diferença só é estatisticamente relevante quando se compara $7^{\circ}$ e $9^{\circ}$ ano.

Os resultados relativos ao z-escore para definição de valores sugestivos de déficits para a amostra aqui pesquisada estão dispostos na Tabela 3. 
Tabela 1. Média, desvio padrão e coeficiente de variação das medidas de fluência de leitura em cada ano escolar

\begin{tabular}{|c|c|c|c|c|c|}
\hline Ano escolar & $\mathrm{n}$ & Descritiva & PPM & PCPM & TTL \\
\hline \multirow[t]{3}{*}{$2^{\circ}$} & 56 & Média & 70,62 & 66,89 & 3,67 \\
\hline & & $d p$ & 25,09 & 24,90 & 1,56 \\
\hline & & cV & 35,53 & 37,23 & 42,51 \\
\hline \multirow[t]{3}{*}{$3^{\circ}$} & 52 & Média & 105,64 & 100,96 & 2,44 \\
\hline & & $d p$ & 32,19 & 31,20 & 0,82 \\
\hline & & CV & 30,47 & 30,90 & 33,61 \\
\hline \multirow[t]{3}{*}{$4^{\circ}$} & 69 & Média & 116,59 & 111,83 & 2,18 \\
\hline & & $d p$ & 31,29 & 31,18 & 0,62 \\
\hline & & CV & 26,84 & 27,88 & 28,44 \\
\hline \multirow[t]{3}{*}{$5^{\circ}$} & 82 & Média & 137,01 & 132,68 & 1,85 \\
\hline & & $d p$ & 27,02 & 26,48 & 0,42 \\
\hline & & CV & 19,72 & 19,96 & 22,70 \\
\hline \multirow[t]{3}{*}{$6^{\circ}$} & 87 & Média & 150,43 & 147,70 & 1,65 \\
\hline & & $d p$ & 22,68 & 23,46 & 0,27 \\
\hline & & CV & 15,08 & 15,88 & 16,36 \\
\hline \multirow[t]{3}{*}{$7^{\circ}$} & 92 & Média & 160,54 & 156,33 & 1,53 \\
\hline & & $d p$ & 22,24 & 24,39 & 0,30 \\
\hline & & CV & 13,85 & 15,60 & 19,61 \\
\hline \multirow[t]{3}{*}{$8^{\circ}$} & 54 & Média & 162,74 & 160,64 & 1,52 \\
\hline & & $d p$ & 26,38 & 26,83 & 0,37 \\
\hline & & CV & 16,21 & 16,70 & 24,34 \\
\hline \multirow[t]{3}{*}{$9^{\circ}$} & 43 & Média & 168,74 & 167,18 & 1,42 \\
\hline & & $d p$ & 13,02 & 13,51 & 0,17 \\
\hline & & CV & 7,72 & 8,08 & 11,97 \\
\hline
\end{tabular}

Legenda: $\mathrm{n}=$ número de alunos; $\mathrm{PPM}=$ palavras por minuto; $\mathrm{PCPM}=$ palavras corretas por minuto; $T \mathrm{TL}=$ tempo total de leitura dp = desvio padrão, $\mathrm{CV}=\mathrm{coeficiente}$ de variação

Tabela 2. Comparação das variações de velocidade de leitura entre os anos escolares por meio do teste t de student bicaudal para amostras não pareadas

\begin{tabular}{ccc}
\hline Comparação & PPM & PCPM \\
\hline $2^{\circ} \times 3^{\circ}$ & 0,000 & 0,000 \\
$3^{\circ} \times 4^{\circ}$ & 0,063 & 0,060 \\
$4^{\circ} \times 5^{\circ}$ & 0,000 & 0,000 \\
$5^{\circ} \times 6^{\circ}$ & 0,001 & 0,000 \\
$6^{\circ} \times 7^{\circ}$ & 0,003 & 0,016 \\
$7^{\circ} \times 8^{\circ}$ & 0,608 & 0,336 \\
$8^{\circ} \times 9^{\circ}$ & 0,147 & 0,122 \\
$7^{\circ} \times 9^{\circ}$ & 0,008 & 0,001 \\
\hline
\end{tabular}

Legenda: Teste t de student com significância de 5\%

Tabela 3. resultados do z-escore para delimitação dos valores sugestivos de déficit para cada ano escolar da presente amostra

\begin{tabular}{|c|c|c|c|c|c|c|c|c|}
\hline & $2^{\circ}$ & $3^{\circ}$ & $4^{\circ}$ & $5^{\circ}$ & $6^{\circ}$ & $7^{\circ}$ & $8^{\circ}$ & $9^{\circ}$ \\
\hline $\begin{array}{l}\text { Sugestivo de déficit } \\
\text { importante }\end{array}$ & $\begin{array}{l}\text { abaixo de } \\
20\end{array}$ & $\begin{array}{l}\text { abaixo de } \\
\quad 47\end{array}$ & $\begin{array}{l}\text { abaixo de } \\
55\end{array}$ & $\begin{array}{l}\text { abaixo de } \\
78\end{array}$ & $\begin{array}{l}\text { abaixo de } \\
93\end{array}$ & $\begin{array}{l}\text { abaixo de } \\
92\end{array}$ & $\begin{array}{l}\text { abaixo de } \\
99\end{array}$ & $\begin{array}{l}\text { abaixo de } \\
108\end{array}$ \\
\hline $\begin{array}{l}\text { Sugestivo de déficit } \\
\text { moderado à severo }\end{array}$ & de 20 a 29 & de 47 a 57 & de 55 a 65 & de 78 a 86 & de 93 a 101 & de 92 a 103 & de 99 a 107 & $\begin{array}{c}\text { de } 108 \mathrm{a} \\
116\end{array}$ \\
\hline Sugestivo de déficit & de 30 a 31 & de 57 a 59 & de 65 a 67 & de 86 a 88 & $\begin{array}{c}\text { de } 101 \text { a } \\
103\end{array}$ & $\begin{array}{c}\text { de } 103 \text { a } \\
105\end{array}$ & $\begin{array}{c}\text { de } 107 \text { a } \\
109\end{array}$ & $\begin{array}{c}\text { de } 116 \text { a } \\
118\end{array}$ \\
\hline $\begin{array}{c}\text { Sugestivo de alerta para } \\
\text { déficit }\end{array}$ & de 32 a 43 & de 59 a 71 & de 67 a 79 & de 88 a 98 & $\begin{array}{c}\text { de } 103 \text { a } \\
113\end{array}$ & $\begin{array}{c}\text { de } 105 \text { a } \\
119\end{array}$ & $\begin{array}{c}\text { de } 109 \text { a } \\
120\end{array}$ & $\begin{array}{c}\text { de } 118 \mathrm{a} \\
128\end{array}$ \\
\hline Não sugestivo de déficit & acima de 43 & acima de 71 & acima de 79 & acima de 98 & $\begin{array}{l}\text { acima de } \\
113\end{array}$ & acima 119 & $\begin{array}{l}\text { acima de } \\
120\end{array}$ & $\begin{array}{c}\text { acima de } \\
128\end{array}$ \\
\hline
\end{tabular}

Legenda: PPM: palavra por minuto; PCPM: palavra correta por minuto; TTL: tempo total de leitura 


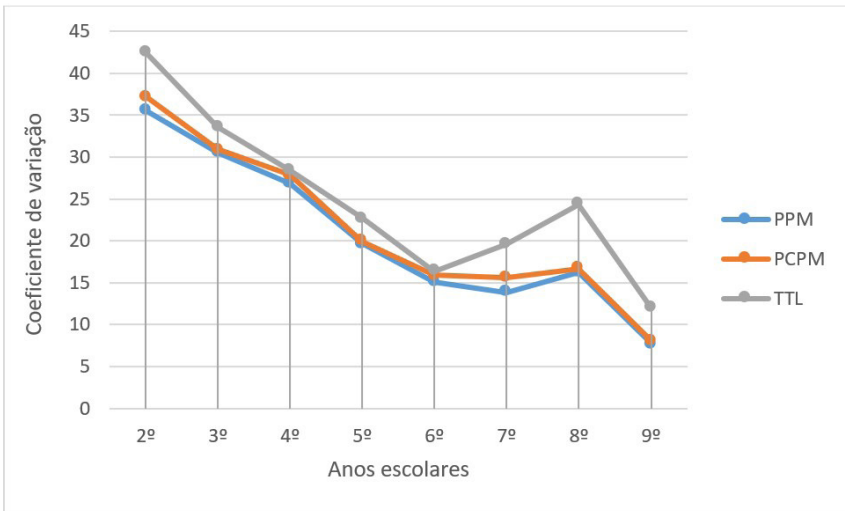

Figura 1. Evolução do coeficiente de variação ao longo dos anos escolares para cada variável de fluência de leitura estudada

\section{DISCUSSÃO}

$\mathrm{Na}$ presente pesquisa optou-se por avaliar a evolução da leitura calculando-se o número de palavras lidas por minuto (PPM) e de palavras lidas corretamente por minuto (PCPM), que são medidas geralmente apontadas como bons marcadores para se acompanhar o desenvolvimento da fluência leitora em estudantes $^{(7,19)}$.

De acordo com os resultados apresentados nos Tabela 1, pode-se observar evolução da leitura realizada pelos alunos do segundo para os alunos do nono ano, marcada pelo aumento do número de PPM e PCPM, assim como diminuição da média do tempo total de leitura dos alunos com o avanço dos anos escolares (Figura 1). Tais resultados nos permitem inferir que o aumento da acurácia possibilita leitura mais rápida, concordando com os achados descritos na literatura, que evidenciam evolução crescente da acurácia de leitura e diminuição no tempo de leitura oral com o avanço do ano escolar ${ }^{(5,12,14,17)}$. É interessante destacar que com o aumento dos anos escolares além do aumento da média de velocidade de leitura e de acurácia, há uma diminuição do coeficiente de variação (Figura 1). Qualitativamente, esses resultados apontam que há uma tendência de homogeneização da amostra estudada: nos anos iniciais os resultados de velocidade de leitura variam mais que nos anos finais do ensino fundamental.

Esses apontamentos qualitativos e quantitativos são também observados quantitativamente: a Tabela 2 indica uma progressão estatisticamente relevante (valor de $\mathrm{p}<0,05$ ) do $2^{\circ}$ ao $7^{\circ}$ ano do Ensino Fundamental, quando PPM e PCPM do ano escolar cursado é comparado com o do ano posterior. A partir do $7^{\circ}$ ano a progressão ainda é observada, porém, de forma mais lenta. A diferença só é estatisticamente relevante quando se compara $7^{\circ}$ e $9^{\circ}$ ano. Ou seja, entre o $2^{\circ}$ e o $7^{\circ}$ ano do Ensino Fundamental, a fluência leitora dos alunos se mostrou em processo de construção, com estabilização a partir do sétimo ano. Por conseguinte, uma leitura mais fluente e homogênea se mostrou sedimentada entre o $7^{\circ}$ e o $9^{\circ}$ ano.

A literatura ${ }^{(3,16)}$ refere que no início da alfabetização a leitura acontece de forma lenta e dispendiosa, pois o alfabetizando utiliza a rota fonológica, estabelecendo o resgate sonoro de cada letra lida, processo exigido para a convenção grafema- fonema. À medida que os escolares adquirem maior contato e se familiarizam com número crescente de palavras escritas de sua língua, passam a realizar leitura utilizando a rota lexical, possibilitando leitura com automaticidade e velocidade cada vez maior.

Um aspecto importante ao se observar a evolução da velocidade de leitura está associado à quantidade de erros cometidos durante essa atividade. Alunos do início do ensino fundamental apresentaram número de PCPM menor que os alunos dos anos finais, o que indica maior quantidade de palavras lidas de maneira errada. Com o aumento do nível de escolaridade, há redução de erros, indicando a apropriação das regras de decodificação ortográfica na leitura, o que possibilita uma leitura mais fluida ${ }^{(24,25)}$. Uma pesquisa norte-americana ${ }^{(19)}$ observou que a precisão (palavras lidas corretamente) é maior para os níveis mais altos de escolaridade, de $70,3 \%$ de palavras lidas corretamente para o primeiro ano com um aumento para $92,6 \%$ e $90,5 \%$ para quinto e sexto anos, respectivamente.

Com o intuito de avaliar a importância da fluência de leitura no desenvolvimento da compreensão da leitura em português nos anos inicias da alfabetização, uma pesquisa brasileira ${ }^{(3)}$ aponta que a habilidade de ler palavras com acurácia e rapidez no final do 10 ano correlacionou-se significativamente com a habilidade de compreensão da leitura ao final do segundo ano do ensino fundamental. O estudo sugere que a fluência contribui significativamente para a compreensão da leitura desde o início da aquisição da leitura ${ }^{(3)}$.

Um estudo consultado ${ }^{(26)}$ considerou que, no percurso do desenvolvimento da leitura, a automaticidade é alcançada quando os leitores atingem cerca de 80 palavras corretas por minuto (PCPM) no texto apropriado à idade, o que indica que o aluno passou do estágio inicial de leitura para um estágio de leitura independente. Embora não haja parâmetros similares para leitores do português brasileiros, observa-se nesta pesquisa que os alunos do terceiro ano do ensino fundamental alcançaram tal média, indicando automaticidade na leitura. Entre o terceiro e o sétimo ano o crescimento continua progressivo e a partir do sétimo tal padrão se estabiliza, uma vez que não há aumento significante na comparação do ganho em fluência de cada ano.

Segundo tal perspectiva, as médias de palavras lidas por minuto do sétimo ao nono ano do Ensino Fundamental podem ser consideradas independentes, estáveis e corroboram os valores encontrados na literatura ${ }^{(25)}$.

À medida que a automaticidade se desenvolve, o desempenho do aluno não apenas se torna preciso, mas também se torna mais rápido. No entanto, esse aumento na velocidade não é ilimitado. Em vez disso, a curva de aprendizado para essas tarefas segue o que é conhecido como power law (lei potencial): o tempo de reação diminui em função da prática até que algum limite irredutível seja alcançado. A velocidade aumenta ao longo da prática, mas os ganhos são maiores no início e diminuem com a prática adicional ${ }^{(10)}$.

Já a escolha de se estudar a fluência de leitura nos anos por meio do z-escore e a proposta de escalonamento ${ }^{(23)}$ se embasou em três pilares principais. Primeiro, para se estudar um caso é importante ter níveis de precisão nos estágios de investigação epidemiológica $^{(27)}$. O uso do z-escore como sugestivo para 
alerta, sugestivo de déficit de moderado à severo ou sugestivo de déficit de gravidade importante é usado em instrumentos pela Fonoaudiologia como pontos de referência ${ }^{(28,29)}$. Em segundo lugar, a proposta permite a visualização de sinais ${ }^{(27)}$, apesar de serem por si só suficientes para conclusão de casos. E por fim, o escalonamento buscou a identificação de casos similares, ou seja, mostra uma tentativa de apresentar uma proposta mais sensível e não específica ${ }^{(27)}$. Com isso, a Tabela 3 permite ao usuário a colocação de um caso individual, dentro de uma sala de aula ou dentro de grupo ou até mesmo consultório.

É importante destacar ainda a proposta de análise de escalas sugestivas para déficit por meio do z-escore apresentada por esta pesquisa. Esses dados, cujos resultados estão apresentados na Tabela 3, mostram uma estratificação desde o déficit importante até o não sugestivo, com um total de cinco estratos. Aqui temos duas colocações para destacar. A primeira diz respeito ao uso metodológico desse tipo de análise. Ao fazer a estratificação, permitimos que os professores e profissionais que lidam com a criança tenham uma referência mais clara sobre qual ponto ela se encontra nas faixas esperadas para a sua escolaridade. Ou seja, é uma planilha que tem como principal objetivo facilitar a análise individual. Por exemplo, se uma criança que está no $2^{\circ}$ ano lê a uma velocidade de 23 palavras por minuto, ela precisa receber atenção especial da escola imediatamente, uma vez que ela apresenta uma velocidade de leitura que indica déficit moderado à severo. Isso mostra o valor particular da tabela: com base nos dados de um grupo, é possível visualizar facilmente o grau de risco daquele escolar dentro dos parâmetros de fluência de leitura.

O segundo ponto importante a se destacar sobre a escala sugestiva para déficit por meio do z-escore é relativo aos dados de base. Dependendo da entrada (de quais foram os escolares que participaram da coleta de dados para análise), a tabela pode variar. Esses resultados não representam o Brasil, representam a amostra coletada para a presente pesquisa. Diferentes contextos podem levar a valores diferentes. Aqui temos um limite da pesquisa: a origem dos dados se limita a uma cidade de Belo Horizonte. Como estratégias para escolas, temos duas possibilidades. A primeira seria um software capaz de realizar essa detecção automaticamente e, dessa forma, realizar a estratificação considerando as particularidades locais. A segunda estratégia, mais dispendiosa, seria realizar a presente pesquisa com alcance nacional, coletando dados, no mínimo, em todos os estados e Distrito Federal.

Os resultados da pesquisa apontaram para evolução da fluência leitora com o avanço dos alunos no processo de escolarização do Ensino Fundamental I para o Ensino Fundamental II, indo ao encontro de estudos que estabelecem relação entre fluência de leitura e avanço no nível de escolarização ${ }^{(6,7,17,19,20,30)}$.

Esta pesquisa se orienta pela necessidade de se estabelecer parâmetros para avaliação da taxa, da acurácia e da fluência de leitura de escolares brasileiros. Por isso, torna-se indispensável pesquisas que investiguem alunos de diferentes sistemas de ensino e regiões do Brasil, além de estender a outros ciclos da educação brasileira, como Ensino Médio e Superior, a fim de se ter um panorama sobre a evolução e mais informações sobre consolidação desta habilidade no sistema de ensino nacional. Também são necessárias pesquisas longitudinais para fornecer informações sobre o surgimento da fluência e como ela se modifica ao longo do tempo, a contribuição de práticas instrucionais específicas, a relação com as séries escolares e como a experiência em leitura impacta o desenvolvimento de uma leitura hábil e eficiente. Como qualquer estudo realizado em uma localidade específica de um país tão grande e extenso como Brasil, os resultados devem ser lidos com cautela para uso em demais localidades.

Outro limite desta pesquisa está nos parâmetros escolhidos para análise. A escolha dos parâmetros temporais exclusivamente, desconsiderando as variações de frequência fundamental, impossibilitam a análise da leitura em grupos tonais. Sugerimos que pesquisas futuras contemplem também a variação melódica em conjunto com os parâmetros de duração para análise da evolução da leitura oral.

\section{CONCLUSÃO}

O estudo apontou evolução da velocidade leitora entre o $2^{\circ}$ e o $7^{\circ}$ ano do ensino fundamental; e do $7^{\circ}$ ao $9^{\circ}$ ano, apesar do aumento observado nas médias, a análise estatística não apontou significância. Tais medidas dos valores esperados para cada ano escolar são essenciais para se conhecer melhor o desenvolvimento da leitura dos escolares, de forma a prover padrões de referência para um adequado monitoramento no âmbito clínico e educacional e predição das habilidades e dificuldades escolares.

Os resultados apontaram uma associação entre o avanço nas séries escolares e as habilidades de leitura. As avaliações de leitura fornecem informações críticas e oportunas para identificar os alunos que precisam de atenção imediata e a velocidade de leitura se mostra uma potencial medida para realizar uma mensuração breve do desempenho e desenvolvimento educacional dos alunos ao longo do avanço escolar. Ressalta-se a necessidade de mais estudos sobre a evolução da leitura, assim como da proposição de parâmetros esperados para cada faixa de escolaridade.

\section{AGRADECIMENTOS}

Agradecemos à Coordenação de Aperfeiçoamento de Pessoal de Nível Superior - Brasil (CAPES) pela disponibilização de materiais científicos, constituindo-se como importante fonte de acesso à informação, que foi utilizada na construção deste estudo. Agradecemos à Fundação de Apoio à Pesquisa do Distrito Federal (FAPDF) pelo apoio financeiro parcial a este artigo.

\section{REFERÊNCIAS}

1. Alves LM, Reis C, Pinheiro AMV. Prosody and reading in dyslexic children. Dyslexia. 2015;21(1):35-49. http://dx.doi.org/10.1002/dys.1485. PMid:25363804

2. Puliezi S, Maluf MR. A fluência e sua importância para a compreensão da leitura. Psico-USF. 2014;19(3):467-75. http://dx.doi.org/10.1590/141382712014019003009.

3. Cardoso-Martins C, Navas AL. O papel da fluência de leitura de palavras no desenvolvimento da compreensão da leitura: um estudo longitudinal: um estudo longitudinal. Educ Rev. 2016;62(62):17-32. http://dx.doi. org/10.1590/0104-4060.48307. 
4. O'Connor RE. Reading fluency and students with reading disabilities: how fast is fast enough to promote reading comprehension? J Learn Disabil. 2018;51(2):124-36. http://dx.doi.org/10.1177/0022219417691835. PMid:29179613.

5. Martins MA, Capellini SA. Relação entre fluência de leitura oral e compreensão de leitura. CoDAS. 2019;31(1):e20170244. http://dx.doi. org/10.1590/2317-1782/20182018244. PMid:30810631.

6. Gentilini LKS, Andrade MEP, Basso FP, Salles JF, Martins-Reis VO, Alves LM. Desenvolvimento de instrumento para avaliação coletiva da fluência e compreensão de leitura textual em escolares do ensino fundamental II. CoDAS. 2020;32(2):e20190015. http://dx.doi.org/10.1590/23171782/20192019015. PMid:32130312.

7. NRP: National Reading Panel. Teaching children to read: an evidencebased assessment of the scientific research literature on reading and its implications for reading instructions. Tecnical Report. USA: National Institute of Child Health \& Human Development, NRP; 2000.

8. Kuhn MR, Schwanenflugel PJ, Meisinger EB, Levy BA, Rasinski TV. Aligning theory and assessment of reading fluency: automaticity, prosody, and definitions of fluency. Read Res Q. 2010;45(2):230-51. http://dx.doi. org/10.1598/RRQ.45.2.4.

9. Hudson RF, Lane HB, Pullen PC. Reading fluency assessment and instruction: what, why, and how? Read Teach. 2005;58(8):702-14. http:// dx.doi.org/10.1598/RT.58.8.1.

10. Logan GD. Automaticity and reading: perspectives from the instance theory of automatization. Read Writ Q. 1997;13(2):123-46. http://dx.doi. org/10.1080/1057356970130203.

11. Geva E, Zadeh ZY. Reading efficiency in native english-speaking and english-as-a-second-language children: the role of oral proficiency and underlying cognitive-linguistic processes. Scientific Studies of Reading. 2006;10(1):31-57. http://dx.doi.org/10.1207/s1532799xssr1001 3 .

12. Kawano CE, Kida ASB, Carvalho CAF, Ávila CRB. Parâmetros de fluência e tipos de erros na leitura de escolares com indicação de dificuldades para ler e escrever. Rev Soc Bras Fonoaudiol. 2011;1(16):9-18. http://dx.doi. org/10.1590/S1516-80342011000100004.

13. Mendonça-Alves L, Latain M, Ghio A, Correa-Celeste L. Escala multidimensional de fluência em leitura: avaliação perceptiva da leitura em escolares com e sem dislexia do desenvolvimento. In: Mousinho R, Alves LM, Capellini S. Dislexia: novos temas, novas perspectivas. Vol. 3. Rio de Janeiro: Wak; 2013. p. 151-164.

14. Celeste LC, Pereira ES, Pereira NRR, Alves LM. Parâmetros prosódicos de leitura em escolares do segundo ao quinto ano do ensino fundamental. CoDAS. 2018;30(1):e20170034. http://dx.doi.org/10.1590/2317-1782/20182017034. PMid:29451669.

15. INEP: Instituto Nacional de Estudos e Pesquisas Educacionais Anísio Teixeira. IDEB - Resultados e Metas. 2017. Consulta ao Índice de Desenvolvimento da Educação Básica [Internet] 2017 [citado em 2019 Ago 1]. Disponível em: http://ideb.inep.gov.br/.

16. Salles JF, Parente MAMP. Avaliação da leitura e da escrita de palavras em crianças de $2^{\circ}$ série abordagem neuropsicológica cognitiva. Psicol Reflex Crit. 2007;2(20):220-8. http://dx.doi.org/10.1590/S0102-79722007000200007.

17. Andrade AJL, Celeste LC, Alves LM. Caracterização da fluência de leitura em escolares do Ensino Fundamental II. Audiol Commun Res. 2019;24:1-8. http://dx.doi.org/10.1590/2317-6431-2018-1983.
18. Fuchs LS, Fuchs D, Hosp MK, Jenkins JR. Oral reading fluency as an indicator of reading competence: a theoretical, empirical, and historical analysis. Sci Stud Read. 2001;5(3):239-56. http://dx.doi.org/10.1207/ S1532799XSSR0503_3.

19. Bolaños D, Cole RA, Ward WH, Tindal GA, Schwanenflugel PJ, Kuhn MR. Automatic assessment of expressive oral reading. Speech Commun. 2013;55(2):221-36. http://dx.doi.org/10.1016/j.specom.2012.08.002.

20. Salles JF, Parente MAMP. Compreensão textual em alunos de segunda e terceira séries: uma abordagem cognitiva. Estud Psicol. 2004;9(1):71-80. http://dx.doi.org/10.1590/S1413-294X2004000100009.

21. IBGE: Instituto Brasileiro de Geografia e Estatística. Censo Escolar, database 2018 [Internet]. 2018 [citado em 2018 Ago 1]. Disponível em: https://cidades.ibge.gov.br/brasil/mg/belo-horizonte/pesquisa/13/5913

22. Boersma P, Weenik D. Praat: doing phonetics by computer. Version 6.0.37 [Internet]. 2020 [citado em 2018 Ago 1]. Disponível em: www.praat.org

23. Schoenberg MR, Dawson KA, Duff K, Patton D, Scott JG, Adams RL. Test performance and classification statistics for the Rey Auditory Verbal Learning Test in selected clinical samples. Arch Clin Neuropsychol. 2006;21(7):693703. http://dx.doi.org/10.1016/j.acn.2006.06.010. PMid:16987634.

24. Avila CR, Kida AS, Carvalho CA, Paolucci JF. Reading errors typology of Brazilian students considered good readers. Pro Fono. 2009;21(4):320-5. http://dx.doi.org/10.1590/S0104-56872009000400010. PMid:20098951.

25. Komeno EM, Ávila CRB, Cintra IP, Schoen TH. Velocidade de leitura e desempenho escolar na última série do ensino fundamental. Estud Psicol. 2015;32(3):437-47. http://dx.doi.org/10.1590/0103-166X2015000300009.

26. Abbott M, Wills H, Greenwood CR, Kamps D, Heitzman-Powell L, Selig J. The combined effects of grade retention and targeted small-group intervention on students' literacy outcomes. Read Writ Q. 2009;26(1):4-25. http://dx.doi.org/10.1080/10573560903396876.

27. Laguardia J, Penna ML. Definição de caso e vigilância epidemiológica. Inf. Epidemiol. Sus. 1999;8(4):63-6. http://dx.doi.org/10.5123/S010416731999000400005.

28. Fonseca RP, de Salles JF, Parente MAMP. Development and content validity of the Brazilian Brief Neuropsychological Assessment Battery Neupsilin. Psychol Neurosci. 2008;1(1):55-62. http://dx.doi.org/10.3922/j. psns.2008.1.009.

29. Bertolucci PH, Brucki SM, Campacci SR, Juliano Y. O mini-exame do estado mental em uma população geral: impacto da escolaridade. Arq Neuropsiquiatr. 1994;52(1):1-7. http://dx.doi.org/10.1590/S0004282X1994000100001. PMid:8002795.

30. Wang C, Algozzine B, Ma W, Porfeli E. Oral reading rates of second-grade students. J Educ Psychol. 2011;103(2):442-54. http://dx.doi.org/10.1037/ a0023029.

\section{Contribuição dos autores}

LMA - responsável pela pesquisa, coleta de dados, análise dos resultados, revisão do estudo e aprovação da versão final a ser publicada. VOMR, LCC e ICCM - responsáveis pela supervisão da execução da pesquisa, análise dos dados, revisão do estudo, aprovação da versão final a ser publicada. LFS - responsável pela análise dos dados e redação do artigo. IMC, GLR e LSCF - responsáveis pelas análises e conferência dos dados e apoio na construção do texto final. 